\title{
Overnight calls in primary care: randomised controlled trial of management using nurse telephone consultation
}

\author{
Felicity Thompson, Steve George, Val Lattimer, Helen Smith, Michael Moore, Joanne Turnbull,
} Mark Mullee, Eileen Thomas, Hugh Bond, Alan Glasper

\begin{abstract}
Health Care
Research Unit Southampton General Hospital, Southampton SO16 6YD

Felicity Thompson researcher

Steve George director

Val Lattimer MRC fellow

Academic Department of Primary Medical Care, Aldemoor Health Centre,

We recently published the results of a randomised controlled trial of a nurse telephone consultation service in primary care out of hours. ${ }^{1}$ The new service, operating at evenings and weekends, significantly reduced general practitioners' workload and was at least as safe as the existing out of hours service. Contacts diminish sharply after about $10 \mathrm{pm},{ }^{2}$ and, anecdotally, a higher proportion of night calls necessitate consultation with a general practitioner. We report here a parallel trial aimed at establishing whether nurse telephone consultation was equally effective in managing workload at night.
\end{abstract} Southampton SO9 $5 \mathrm{NH}$

Helen Smith senior lecturer Michael Moore general practitioner Hugh Bond general practitioner

University Department of Medical Statistics, Southampton General Hospita Joanne Turnbull data manager Mark Mullee senior research fellow

School of

Postgraduate Medicine, Queen Alexandra Hospital, Portsmouth, PO6 3LY

Eileen Thomas reader in health studies

School of Nursing, Southampton SO17 1BJ

Alan Glasper professor

Correspondence to: Dr George pluto@soton.ac.uk

BMJ 1999;319:1408

\section{Subjects, methods, and results}

This study was an adjunct to a randomised controlled trial in a 55 member general practice cooperative serving 97000 patients in Wiltshire. The design has been described. ${ }^{1}$ The night nurse telephone consultation service ran over two two-week periods (15-28 October 1997 and 12-25 November 1997) from 11.15 pm until $8 \mathrm{am}$. Outcome measures were as used in the main trial with one addition: the number of patients attending daytime surgery within three days of a call. ${ }^{1}$ One of us (FT) visited each surgery to extract details of attendances from patient records.

In the main study $49.8 \%$ of calls were handled by the nurse alone. Specifying $\alpha=0.1$ ( 0.05 in a one sided calculation) and $\beta=0.2$, we calculated that the night nurse service would need to receive 78 calls to establish equivalence with this figure, with equivalence limits being $40 \%$ and $60 \% .{ }^{3}$ A one sided calculation was used as we were interested to establish only whether the night nurse intervention produced worse results (lower numbers of calls handled without referral to a doctor) than the evening and weekend service. For other within-trial outcomes, results are presented as relative risks with 95\% confidence intervals, calculated with EpiInfo. This trial was not powered to show within-trial equivalence in numbers of adverse events.

Number (\%) of calls at night, by trial group, showing management outcome and relative risk ( $95 \%$ confidence interval) for differences between groups

\begin{tabular}{lccc} 
Management outcome & $\begin{array}{c}\text { Intervention } \\
\text { group }\end{array}$ & $\begin{array}{c}\text { Control } \\
\text { group }\end{array}$ & Relative risk (95\% CI) \\
\hline Total No of calls & $100(100)$ & $123(100)$ & NA \\
\hline Calls managed with nurse telephone advice & $59(59)$ & NA & NA \\
\hline Calls managed with GP telephone advice & $19(19)$ & $76(62)$ & 0.31 (0.2 to 0.47) \\
\hline Patient attended a primary care centre & $1(1)$ & $6(5)$ & 0.2 (0.03 to 1.67) \\
\hline Patient were visited at home by duty GP & $21(21)$ & $41(33)$ & 0.63 (0.4 to 0.99) \\
\hline Patient died within 7 days & $2(2)$ & $2(2)$ & 1.23 (0.18 to 8.58) \\
\hline Patient admitted to hospital within 24 hours & $2(2)$ & $8(6.5)$ & 0.31 (0.07 to 1.42) \\
\hline Patient admitted to hospital within 3 days & $5(5)$ & $8(6.5)$ & 0.77 (0.26 to 2.28) \\
\hline Patient attended A\&E department within 3 days & $3(3)$ & $2(2)$ & 1.84 (0.31 to 10.82) \\
\hline Patient attended daytime surgery within 3 days & $8(8)$ & $18(15)$ & 0.55 (0.25 to 1.2) \\
\hline
\end{tabular}

$\mathrm{NA}=$ not applicable; $\mathrm{GP}=$ general practitioner; $\mathrm{A} \& \mathrm{E}=$ accident and emergency.
During the study 210 callers made 223 calls, 123 in the control group and 100 in the nurse telephone consultation (intervention) group. Follow up was $94 \%$ complete: 12 sets of patient records $(6 \%)$ could not be found, seven in the control group and five in the intervention group. The median age (range) of patients was $34.0(0.01-97.2)$ years in the control group and 32.5 $(0.49-97.0)$ years in the intervention group. Fifty three patients (43\%) in the control group and $44(44 \%)$ in the intervention group were male.

The table shows details of call management and outcome. Altogether 59\% of calls (95\% confidence interval $48.7 \%$ to $68.7 \%$ ) were handled by the nurse alone. As we were interested only in whether the nurse service handled fewer calls at night, this can be interpreted as showing equivalence with the proportion observed in the main trial. The proportions of calls in which callers received advice from a general practitioner and calls ending in a home visit showed clear reductions, with $95 \%$ confidence intervals not embracing 1. A lower proportion of calls resulted in a daytime surgery attendance in the intervention arm, although the 95\% confidence interval embraced 1 . Other differences had wide confidence intervals.

\section{Comment}

This study shows that nurses on the telephone can manage as high a proportion of primary care calls at night as during evenings and weekends, and without more patients attending daytime surgery within the next three days. Over the same period as this study, however, the evening and weekend service received 994 calls-over four times as many as at night, and in fewer hours. A nurse telephone consultation service at cooperative level might therefore be uneconomic at night. In that case the economies of scale offered by larger groups of practices, or by NHS Direct, may be beneficial. ${ }^{4}$

We thank the Royal College of Nursing for its support, and Dr Jeremy Dale and Mr Robert Crouch, of King's College Hospital, London, and Mr Mike Bennett, of Plain Software, for their help.

Contributors: SG, VL, HS, ET, and EAG initiated the study and obtained funding. M Moore and FT facilitated the piloting; VL, FT, M Moore, and HB were responsible for running the service. Data collection was done by VL, FT, and JT and data analysis by FT, JT, M Mullee and SG. All authors participated in the discussion about and interpretation of the results. FT and SG wrote the paper, with comments from all authors. SG is the guarantor.

Funding: British Telecom and the South and West regional office of the NHS Executive funded this work.

Competing interests: None declared.

1 Lattimer V, George S, Thompson F, Thomas E, Mullee M, Turnbull J, et al. Safety and effectiveness of nurse telephone consultation in out-of-hours primary care: randomised controlled trial. BMJ 1998;317:1054-9.

2 Brogan C, Pickard D, Gray A, Fairman S, Hill A. The use of out of hours health services: a cross sectional survey. BMJ 1998;316:524-7.

3 Jones B, Jarvis P, Lewis JA, Ebbutt AF. Trials to assess equivalence: the importance of rigorous methods. BMJ 1996;313:36-9.

4 College of Health. Developing NHS Direct. London: $\mathrm{CoH}, 1998$. (Accepted 29 July 1999) 


\title{
Comparison of stool immunoassay with standard methods for detecting Helicobacter pylori infection
}

\author{
Frank Lehmann, Jürgen Drewe, Luigi Terracciano, Robert Stuber, Reno Frei, Christoph Beglinger
}

Helicobacter pylori is the cause of type B gastritis and associated with peptic ulcer disease. Various methods are available for detecting $H$ pylori, but all have limitations. ${ }^{1} H$ pylori infection can be diagnosed by tests requiring endoscopy (rapid urease test, histology, culture) and by non-invasive tests (carbon-13 urea breath test, serology, stool tests). The urea breath test is currently the most important test for follow up after $H$ pylori treatment. ${ }^{1}$ Serology is widely used for screening patients for $H$ pylori infection; it has a good sensitivity, is fast, and relatively inexpensive. ${ }^{1}$ However, the urea breath test is expensive and requires specialised equipment, and serological tests cannot be used after $H$ pylori treatment and may have a lower specificity.

Most patients infected with $H$ pylori are treated by general practitioners, who need an easy test for it. Recently, an immunoassay has been developed that can detect $H$ pylori antigen in human faeces, ${ }^{2}$ but it has not been validated for clinical use. We studied patients undergoing routine endoscopy to determine sensitivity and specificity of this immunoassay in comparison with standard methods.

\section{Subjects, methods, and results}

We recruited 102 consecutive patients (58 men, 44 women) undergoing upper endoscopy. Our study was approved by the local ethics committee. We determined $H$ pylori status in all patients by rapid urease test, histology, and culture using standard methods. ${ }^{1}$ Patients were considered positive if at least two of the three tests were positive. Patients were asked to collect a specimen from their first stool after endoscopy and to post it to us. We analysed the stool specimens for $H$ pylori antigen using the Premier Platinum HpSA Immunoassay as described by the manufacturer (Meridian Diagnostics, Cincinnati, $\mathrm{OH}$, USA). The test is based on a capture of polyclonal antibodies to $H$ pylori adsorbed to microwells. The results were analysed by spectrophotometric determination and considered positive if the optical density was $>0.12$ and negative if it was $<0.10$. We calculated the test's sensitivity, specificity, and positive and negative predictive values.

Forty nine of the patients had dyspepsia, 33 had active ulcer disease, 16 had gastric or duodenal erosions, and four had gastric polyps. The table shows the results of the immunoassay compared with the standard tests: two stool tests were classified as false negative and four as false positive. The immunoassay

Detection of $H$ pylori infection by stool immunoassay in comparison with standard tests (histology, rapid urease test, and bacteriology). Values are numbers of tests

\begin{tabular}{lcc} 
& \multicolumn{3}{c}{ Standard tests } \\
& H pylori positive $(\mathbf{n}=\mathbf{5 0})$ & H pylori negative $(\mathbf{n}=\mathbf{5 2})$ \\
\hline Stool immunoassay: & & \\
\hline Positive & 48 & 4 \\
\hline Negative & 2 & 48 \\
\hline
\end{tabular}

thus had a sensitivity of $96 \%(95 \%$ confidence interval $90.6 \%$ to $100 \%)$, specificity of $93 \%(85.1 \%$ to $99.5 \%)$, positive predictive value of $92 \%$, and negative predictive value of $96 \%{ }^{3}$

\section{Comment}

Our study indicates that this immunoassay could be used as a routine diagnostic tool for $H$ pylor $i$ infection. It seems to overcome some limitations of previous tests. We found a high sensitivity and specificity compared withreference tests. This newimmunoassay has theadvantage of being non-invasive, easy and fast to perform, and cheaper than the urea breath test. The bacterium does not need to be alive; preliminary data suggest that the test can be used even during $H$ pylori treatment. ${ }^{4}$

The new immunoassay seems to meet the requirements of general practitioners, who treat most patients infected with $H$ pylori, because it is easy to perform, requires no blood samples to be taken, and its costs are similar to those of serological tests. Other stool tests have been developed, ${ }^{5}$ but they cannot be used for clinical practice, either because they require specialised equipment (culture, polymerase chain reaction, etc) or because they have not been validated for clinical use (immunoassay).

This stool immunoassay represents a new, accurate, and non-invasive method for $H$ pylori infection that overcomes the limitations of existing tests.

Contributors: FL coordinated the study, collected samples, and helped with data analysis. JD performed the statistical analysis. LT made the histological assessments. RS and RF helped with collecting samples and supervised and performed the stool analyses. The paper was jointly written by FL, RF, and $\mathrm{CB}$, who had the original idea for the study. CB is guarantor for the study. Competing interests: None declared.

1 Mégraud F. The most important diagnostic modalities for Helicobacte pylori, now and in the future. Eur $J$ Gastroenterol Hepatol 1997;9(suppl): $13-5$.

2 Thomas JE, Gibson GR, Darboe MK, Dale A, Weaver LT. Isolation of Helicobacter pylori from human faeces. Lancet 1992;340:1194-5.

3 Simel DL, Samsa GP, Matchar DB. Likelihood ratios with confidence: sample size estimation for diagnostic test studies. J Clin Epidemiol 1991;44:763-70.

4 Vaira D, Malfertheiner P, Mégraud F, Axon A, Deltenre M, Dixon M, et al. A novel antigen assay based on stool specimen for Helicobacter pylori European multicenter study. Gut 1998;43(suppl 2):A47.

5 Cutler AF, Havstad S, Ma CK, Blaser MJ, Perez-Perez GI, Schubert TT Accuracy of invasive and noninvasive tests to diagnose Helicobacter pylori infection. Gastroenterology 1995;109:136-41.

(Accepted 23 August 1999)

\section{Endpiece}

\section{On ageing: oh dear!}

Youth is a blunder; manhood a struggle; old age a regret.

Coningsby, Benjamin Disraeli, 1804-81

Submitted by Fred Charatan, retired geriatric psychiatrist, Florida
Division of

Gastroenterology,

University Hospital,

CH-4031 Basle

Switzerland

Frank Lehmann

consultant in

gastroenterology

Christoph

Beglinger

professor of medicine

Department of

Clinical

Pharmacology,

University Hospital,

Basle

Jürgen Drewe

associate professor

Department of

Bacteriology,

Central Laboratory,

University Hospital,

Basle

Reno Frei

head of bacteriology

laboratory

Robert Stuber

consultant in

laboratory analyses

Department of

Pathology,

University Hospital,

Basle

Luigi Terracciano

head of

gastrointestinal

pathology

Correspondence to:

C Beglinger

beglinger@tmr.ch

BMJ 1999;319:1409 